\title{
Lesch Nyhan Syndrome
}

\author{
K. M. D. L. Siriwardena \\ West China School of Medicine, Sichuan University, Chengdu, Sichuan, P.R.China. \\ 1955063332@qq.com
}

\begin{abstract}
Lesch Nyhan syndrome (LNS) is a rare genetic disorder at the chromosome point Xq26-q27.2. This disease occurs almost exclusively in male children at a very young age and caused by mutations in the hypoxanthine-guanine phosphoribosyl transferase (HPRT) gene and is inherited in an X-linked recessive manner. The clinical manifestations are associated with the hypoxanthine-guanine phosphoribosyl transferase (HPRT) enzyme deficiency. The symptoms of LNS may become apparent as early as six months of age. The first clinical manifestation of LNS is earlier urate crystal formation, resulting from abnormally elevated levels of uric acid in the urine, leads to the presence of orange colored deposits in the diapers of affected infants. Self-mutilation, mental retardation, self injurious behavior, gouty arthritis, hemiplegia and kidney stones may commonly occur in later stages. LNS is diagnosed by hyperuricemia, macrocytic anaemia, HPRT enzyme activity, identification of a molecular genetic mutation in the HPRT gene, imaging studies of the brain and spinal cord, ultrasound imaging of the urinary tract and slightly delayed bone age. Although treatment of the condition is limited; management of symptoms is effective for most individuals for surviving into the second or third decade of life. Hence there may be slow progression of disease in adulthood; sudden death may highly occur with respiratory abnormalities.
\end{abstract}

My review may provide the latest clinical information and findings of Lesch Nyhan syndrome. Since this disease is rare in the society, researches and information sources are also limited. But this information provided here may help to find some interesting pathways for new research and findings on LNS.

Keywords: Lesch Nyhan syndrome (LNS), Choreoathetosis, X-linked hyperuricemia, Hypoxanthine-guanine phosphoribosyl transferase (HPRT) enzyme deficiency, X-linked hereditary disorder, Self-mutilation syndrome

\section{AbBreviations}

LNV - Lesch Nyhan Variants

LNS - Lesch Nyhan syndrome

HPRT - Hypoxanthine-guanine Phosphoribosyl Transferase

LND - Lesch Nyhan Disease

HPO - Human Phenotype Ontology

ESRD - End-stage renal disease

\section{INTRODUCTION}

Lesch-Nyhan syndrome (LNS) also known as choreoathetosis self-mutilation syndrome and X-linked hyperuricemia is a rare hereditary disorder characterized by neurological and behavioral abnormalities and overproduction of uric acid in the body. Signs and symptoms may include inflammatory arthritis (gout), kidney stones, bladder stones, and moderate cognitive disability. Nervous system and behavioral disturbances also occur, such as involuntary muscle movements and self injury (including biting and head banging). These clinical manifestations are a spectrum associated with the enzyme hypoxanthine-guanine phosphoribosyl transferase (HPRT) deficiency. At one end of the spectrum are patients with classic LNS and the full clinical phenotype. At 
the other end of the spectrum are patients with overproduction of uric acid but no apparent neurological or behavioral deficits (Lesch Nyhan Variants, LNV) - eg: Kelley-Seegmiller syndrome. In between are patients with varying degrees of motor, cognitive, or behavioral abnormalities.

This disease occurs almost exclusively in male children at a very young age and caused by changes (mutations) in the HPRT1 gene and is inherited in an X-linked recessive manner. Treatment is symptomatic and supportive.

This review is about general medical information of Lesch Nyhan Syndrome and its latest diagnosing and treatment methods. Since this disease is very rare the researches and findings sources are limited. However the knowledge reported in this article will help to get a clear diagnosis pathway about LNS.

\section{EPIDEMIOLOGY}

LNS is a rare hereditary disorder. As a X-linked recessive disorder it affects almost exclusively males, with female cases being a rarity.

\section{HISTORY}

The condition was described by Lesch and Nyhan in 1964. The hypoxanthine-guanine phosphoribosyl transferase (HPRT) enzymatic defect which cause LNS was discovered by Seegmiller and colleagues in 1967 and the gene encoding the enzyme was cloned and sequenced by Friedmann and colleagues in 1985.

\section{PATHOPHYSIOLOGY}

LNS is a X-linked condition with a defect at the chromosome point Xq26-q27.2. There is virtually no HPRT in LNS, with levels below $1.5 \%$ but in Kelley-Seegmiller syndrome, levels are at least $8 \%$. Whereas chromosome point Xq26-q27.2 is involved in both disorders, the exact point of mutation varies, even within LNS, and with its clinical manifestation.

In a population at equilibrium for a sex-linked lethal genetic abnormality, one third of the genes would be expected to arise by spontaneous mutation. Since LNS etiology involves a mutation of the HPRT gene, which is on the long arm of the X chromosome (Xq26.1), and it contains 9 exons and 8 introns. Because the HPRT gene is on the $\mathrm{X}$-chromosome, males are affected and females in the families are at risk of being carriers of the mutation. Although female carriers are usually asymptomatic, they may have increased uric acid excretion and some may develop symptoms of hyperuricemia in later years.

When an affected male is the only affected individual in the family, several possibilities regarding his mother's carrier status need to be considered: The son has a de novo pathogenic variant in HPRT1 and his mother is not a carrier, His mother has a de novo pathogenic variant in HPRT1, either as a germline variant (occurring at the time of her conception) or as germline mosaicism (occurring post-fertilization) or His mother has a pathogenic variant that she inherited from a maternal female ancestor.

Many mutations responsible for either full (classic LND) or partial (LNV, Lesch-Nyhan variants) deficiency of HPRT have been identified. Summaries of the different mutation categories appear in the table (Table 1) below. Every individual mutation in each category is listed in separate.

Table1. Mutations of HPRT gene

\begin{tabular}{|c|c|c|c|c|}
\hline All mutations & LND & LNV & NA & Total \\
\hline Single base substitutions & 255 & 121 & 5 & 381 \\
\hline Deletions & 146 & 6 & 6 & 158 \\
\hline Duplications & 37 & 3 & 0 & 40 \\
\hline Other mutations & 23 & 12 & 1 & 36 \\
\hline Total & 461 & 142 & 12 & 615 \\
\hline
\end{tabular}

American Research Journal of Pediatrics

Page 2 
Lesch Nyhan Syndrome

\begin{tabular}{|c|c|c|c|c|}
\hline Single base substitutions & LND & LNV & NA & Total \\
\hline Missense & 131 & 107 & 4 & 242 \\
\hline Nonsense & 47 & 1 & 1 & 49 \\
\hline Splice error & 77 & 13 & 0 & 90 \\
\hline Total & 255 & 121 & 5 & 381 \\
\hline Deletions & LND & LNV & NA & Total \\
\hline Coding sequences & 138 & 5 & 5 & 148 \\
\hline Splice error & 8 & 1 & 1 & 10 \\
\hline Total & 146 & 6 & 6 & 158 \\
\hline
\end{tabular}

\begin{tabular}{|c|c|c|c|c|}
\hline Duplications & LND & LNV & NA & Total \\
\hline Coding sequences & 35 & 3 & 0 & 38 \\
\hline Splice error & 2 & 0 & 0 & 2 \\
\hline Total & 37 & 3 & 0 & 40 \\
\hline
\end{tabular}

\begin{tabular}{|c|c|c|c|c|}
\hline Other mutations & LND & LNV & NA & Total \\
\hline Translocation & 1 & 0 & 0 & 1 \\
\hline Regulatory [1] & 2 & 4 & 0 & 6 \\
\hline Double mutation & 0 & 2 & 0 & 2 \\
\hline Insertation & 3 & 1 & 0 & 13 \\
\hline Complex deletion & 9 & 3 & 1 & 10 \\
\hline Females [2] & 8 & 2 & 0 & 36 \\
\hline Total & 23 & 12 & 1 & 4 \\
\hline
\end{tabular}

(LND-Lesch Nyhan Disease; LNV-Lesch Nyhan variants; NA-Mutations not belongs to LND AND LNV)

\section{SIGNS AND SYMPTOMS}

The following table (Table 2) lists symptoms which vary from each LNS patient. This information comes from a database called the Human Phenotype Ontology (HPO).

Table2. Common symptoms of LNS affected population

\begin{tabular}{|c|l|}
\hline LNS Affected population (\%) & \multicolumn{1}{|c|}{ Common symptoms } \\
\hline $80 \%-99 \%$ & Behavioral abnormality \\
& Gout \\
& Hemiplegia/hemiparesis \\
& Intellectual disability, mild \\
& Intellectual disability, moderate \\
& Spasticity \\
\hline $30 \%-79 \%$ & Anemia \\
& Hematuria \\
& Renal insufficiency \\
\hline
\end{tabular}


Lesch Nyhan Syndrome

\begin{tabular}{|l|l|}
\hline$<30 \%$ & Abnormality of extrapyramidal motor function \\
& Choreoarthritis \\
& Dysarthria \\
Dysphagia & Dystonia \\
& Generalized hypotonia \\
& Hyperreflexia \\
& Hyperurocosuria \\
& Intellectual disability \\
& Megaloblastic anemia \\
& Motor delay \\
& Nephrolithiasis \\
& Opisthotonus \\
& Podagra \\
& Short stature \\
& Testicular atrophy \\
& Vomiting \\
& X-linked recessive inheritance \\
\hline
\end{tabular}

According to the table shown above LNS symptoms are mainly affected on urinary, brain and nervous, skeletal, reproductive system, and so on. They are described in details below.

\section{Urinary System}

The symptoms of LNS may become apparent as early as six months of age. Earlier urate crystal formation, resulting from abnormally elevated levels of uric acid in the urine, leads to the presence of orange colored deposits ("orange sand") in the diapers of infants with this disorder. This may be the first clinical manifestation of Lesch Nyhan syndrome, but it is not often recognized diagnostically in early infancy.

Urate stones may develop in the kidneys of infants with LNS as a result of excessive amounts of uric acid that are excreted as sodium urate. These stones may induce blood to appear in the urine (hematuria) and increase the risk of urinary tract infections.

End-stage renal disease (ESRD) may occur, which prior to the availability of allopurinol, is less common now but still can be occurred.

\section{Brain and Nervous System}

Individuals with LNS typically have a normal prenatal and perinatal course. Neurological symptoms associated with LNS usually begin before the age of 12 months.

They may also have symptom such as involuntary writhing movements of the arms and legs (dystonia) as well as purposeless repetitive movements (chorea) such as flexing of the fingers, raising and lowering of the shoulders, and/or facial grimacing and sometimes ballismus.

Infants who had previously been able to sit upright generally lose this ability. Initially, muscles may be soften (hypotonia) and lead to difficulty in holding the head in an upright position.

Affected infants may fail to reach general developmental milestones such as crawling, sitting or walking (developmental delay). Eventually, they also develop signs of pyramidal involvement including abnormally increased muscle tone (hypertonia), muscle rigidity (spasticity) and deep tendon reflexes are increased (hyperreflexia). The neurologic symptoms closely resemble athetoid cerebral palsy. Mental retardation may 
also occur progressively. General learning difficulties are usual and IQ is typically around 60. However, accurate evaluation of intelligence may be difficult because of poorly articulated speech (dysarthria), and some patients have demonstrably normal cognitive function as well.

The most striking cognitive and behavioral disturbance of LNS, which has been observed in majority of cases, is self-mutilation. These behaviors most commonly begin between the ages of two and three years. However, they also can similarly develop during the first year of life or much later during childhood. Self-injurious behavior may consist of repeated biting of the lips, fingers, and/or hands, and repetitive banging of the head against hard objects. Some children may scratch their face constantly, serious injuries or scarring from repetitive selfabrasion or hitting is seen. Although individuals with LNS are not insensitive to pain, additional behavioral abnormalities may include aggressiveness, vomiting, and spitting. Self-mutilating behaviors often lead to loss of tissue.

Children with LNS may have difficulty swallowing (dysphagia) and may be difficult to feed. Most affected children are underweight for their age. Additionally may include symptoms such as irritability or screaming. Some children with LNS may also develop a rare anemia known as megaloblastic anemia which is unresponsive to vitamin supplements.

Additionally symptoms of LNS may be severe muscle spasm that causes the back to arch severely and the head and heels to bend backward (opisthotonos). Affected children may also experience hip dislocation, fractures, abnormal curvature of the spine (scoliosis) and/or permanent fixation of several joints in a flexed position (contractures).

Imaging findings of patients with classic Lesch Nyhan disease had a 20\% reduction in intracranial volume. ( $17 \%$ reduction in grey matter volume) Classic disease showed additional grey matter volume reductions in the temporal lobe and left lateralized structures, and patients with variant disease showed additional reductions in lingual and precuneus regions with sparing of right frontal and temporal regions and $26 \%$ reduction in white matter volume compared with healthy population. Patients with classic disease had reductions of volume in the ventral striatum and prefrontal areas compared with those with the variant form.

The brain volume abnormalities were not associated with enlarged ventricles or sulci, or with decreased ratios of brain volume to intracranial volume. CT scans have been reported, evidence of cerebral atrophy and enlarged Sylvian fissures in some cases. In some cases of LNS, MRI reported moderate cerebral atrophy, and decreased T2 signal in the basal ganglia. Parenchymal volume loss, focal bifrontal atrophy, brainstem atrophy, manifested by prominence of the prepontine cistern and other basilar cisterns. Prominent thickening of the skull marrow and abnormal T1 signal within the marrow was isointense to brain. Low basal ganglia volume reduced brain volume, particularly in caudate, increases in ventricular size and mild cortical atrophy and/ or brainstem atrophy. Reduced total cerebral volume and reduced volumes in the caudate and putamen also reported in some cases.

The reduction in brain size was accompanied by thickened calvaria and increased sinus pneumatization or sinus size. The increased thickness of the skull and in sinus size may reflect a failure of continued brain growth, probably beginning in infancy.

Less commonly noted findings have in cluded demyelinative lesions, cerebral edema, marked hydrocephalus, and periodic acid-Schiff positive inclusions in the olivary nuclei.

\section{Skeletal System}

Progressively urate crystals may be found in the joints, but in common, it is not until late teenager-hood or adulthood that untreated patients with Lesch Nyhan syndrome experience recurring episodes of pain, tenderness, redness and swelling of the joints, which is commonly known as gouty arthritis. These episodes may become progressively more often once they begin. 
In older children with this disorder, deposits of sodium urate may collect in cartilaginous tissues in joints and in the ears by forming visible "bulges" called tophi in ear. This is also a common condition in gout.

Although female carriers usually do not have symptoms of the disorder, may develop gout later in life as a result of untreated excess uric acid in the blood (hyperuricemia).

\section{Reproductive System}

Delayed puberty, Affected males may have testicular atrophy.

\section{COMPLiCATIONS}

There may be quite severe self-injuries, complications from the neuromuscular imbalance, problems of stones in the renal tract and joint manifestations of gout.

\section{Diagnosis}

\section{Hyperuricemia}

Hyperuricuria nor hyperuricemia (serum uric acid concentration $>8 \mathrm{mg} / \mathrm{dL}$ ) is sensitive and specific enough for diagnosis. Additionally urinary urate-to-creatinine ratio greater than 2.0 may also indicate uric acid overproduction.

Uric acid levels (in blood and urine) are usually raised but can fall transiently into the normal range and can be misled. Urinary excretion of uric acid, especially if corrected for weight, is elevated but collecting a 24-hour sample from an infant is very difficult and a spot test may be unsatisfactory.

\section{Macrocytic Anaemia}

There is often a macrocytic anaemia, although folate and vitamin B12 levels are normal, as is iron. The problem appears to be reduced ability to use vitamin B12.

\section{HPRT Enzyme Activity}

Definitive diagnosis is often by measurement of HPRT enzyme activity(less than $1.5 \%$ of normal) in blood or tissue. Blood samples are often used but intact fibroblasts or lymphocytes give more precise information.

\section{Identification of a Molecular Genetic Mutation in the HPRT Gene}

Genetic mutations in HRPT gene may confirm the diagnosis. This permits carrier detection and prenatal screening of at-risk pregnancies.

- Molecular genetic testing: Once the HPRT1 pathogenic variant has been identified in an affected family member, prenatal testing for a pregnancy at higher risk and preimplantation genetic diagnosis for LeschNyhan syndrome are possible.

- Biochemical genetic testing: Assay of HPRT enzyme activity in cultured amniocytes or chorionic villus cells is the preferred method for prenatal testing if the HPRT1 pathogenic variant has not been identified in the family. No false positive or false negative diagnoses have been encountered in prenatal testing but it is possible that maternal cells could contaminate and overgrow the culture.

In view of the possibility of genetic mosaicism, gene testing can be performed before pregnancy to assess risk and also to assess more distant relatives. Hair roots may be used for this purpose. This may be followed by chorionic villus sampling and, ultimately, termination of the pregnancy. 
Hence LNS is inherited disease, family planning is an important issue in this population. Therefore the optimal time for determination of genetic risk, clarification of carrier status, and discussion of the availability of prenatal testing is before pregnancy. Furthermore it is appropriate to offer genetic counseling (including discussion of potential risks to offspring and reproductive options) to young adults who are affected, are carriers, or are at risk of being carriers.

DNA banking is the storage of DNA (typically extracted from white blood cells) for possible future use. Because it is likely that testing methodology and the understanding of genes, allelic variants, and diseases will improve in the future, consideration should be given to banking DNA of affected individuals.

Imaging studies of the brain and spinal cord.

\section{Ultrasound Imaging of the Urinary Tract}

This examination is important especially as urate stones are radiolucent. It may be conducted periodically in case of stones or obstruction of the urinary tract.

\section{Slightly Delayed Bone Age}

It's usually done by taking a single X-ray of the left wrist, hand and fingers.

\section{MANAGEMENT}

Treatment of the condition is limited:

\section{Treatments for Hyperuricaemia}

Allopurinol can be used for hyperuricaemia and reduces complications (nephrolithiasis and gouty arthritis). Maintaining good hydration helps but despite these measures nephrolithiasis still occurs as well.

\section{Treatments for Spasticity}

Combination of baclofen and benzodiazepines can be used. Diazepam is most commonly used. They may help some of the extrapyramidal features as well.

\section{Treatments for Behavioral Abnormalities}

It is generally managed by using a behavioral modification technique together with medication. It works on the basis of reward or reinforcement of good behavior. Punishment or negative reinforcement of bad behavior may exacerbate problems. Gabapentin and carbamazepine may help reduce some of the behavioral problems.

\section{Treatments to Control Self-Injury by Biting}

At the condition of other techniques fail to control self-injury by biting, dental extraction may be required. Biting does seem to be especially difficult to control. Use of restraints, especially at night, may be justified, even long-term, to prevent self-injury.

Other relevant surgery may be required for muscular and joint problems due to the neurological problem.

Intervention may be required for stones in the urinary tract.

\section{PROGNOSIS}

If management of symptoms is effective, most individuals survive into the second or third decade of life. There may be slow progression of disease in adulthood. 
Sudden death is highly being recognized. Respiratory abnormalities have been implicated. Death is usually from aspiration pneumonia or complications of nephrolithiasis. Sudden death appears to be more common in older individuals, often with unknown cause.

\section{CONCLUSION}

LNS is a rare X-linked recessive disorder which affects almost exclusively males, with female cases being a rarity. The etiology of this condition may involve a mutation of the HPRT gene, which is on the long arm of the X chromosome (Xq26.1), and it contains 9 exons and 8 introns. Most common signs and symptoms are over production of uric acid, neurologic dysfunctions, cognitive and behavioral disturbances, gouty arthritis. Hyperuricemia, macrocytic anaemia, measurement of HPRT enzyme, identification of a molecular genetic mutation in the HPRT gene may help with the diagnosis of LNS. Imaging studies of the brain and spinal cord also tend to show some specific abnormalities for diagnosis.

Hence treatment of the condition is limited; management of symptoms is effective for most individuals survive into the second or third decade of life. Al though there may be slow progression of disease in adulthood, sudden death is highly being recognized by respiratory abnormalities and in older individuals often with an unknown cause.

Purpose of this review is to provide the latest clinical information of the LNS and searching current findings and research directions as well. Since this disease is rare in the society, researches and information sources are also limited. But this article would help to find some interesting pathways for new research and findings on LNS.

\section{REFERENCES}

1. James C. Harris, MD; Roland R. Lee, MD; Hyder A. Jinnah, MD, PhD; et al, Craniocerebral Magnetic Resonance Imaging Measurement and Findings in Lesch-Nyhan Syndrome. Arch Neurol. 1998; 55(4):547-553.

2. Khue Vu Nguyen, Epigenetic regulation in amyloid precursor protein and the Lesch Nyhan syndrome, Biochemical and biophysical research communications 446 (2014) 1091-1095.

3. Yoshiaki Saito a,b,c, Sachio Takashima a,b, Neurotransmitter changes in the pathophysiology of Lesch \pm Nyhan syndrome, Brain \& Development 22 (2000) S122-S131

4. David J Schretlen, Mark Varvaris, Tiff any E Ho, Tracy D Vannorsdall, Barry Gordon, James C Harris, H A Jinnah, Regional brain volume abnormalities in Lesch-Nyhan disease and its variants: a cross-sectional study, Lancet Neurol 2013; 12: 1151-58

5. Kyriakie Sarafoglou, MD; Krista Grosse-Redlinger, CGC; Christopher J. Boys, PhD; et al Laurence Charnas, MD; Noelle Otten, MS; Robyn Broock, PhD; William L. Nyhan, MD, PhD, Lesch-Nyhan Variant SyndromeVariable Presentation in 3 Affected Family Members, Copyright 2010 American Medical Association. All Rights Reserved.Applicable FARS/DFARS Restrictions Apply to GovernmentUse.2010 Arch Neurol.2010;67(6):761764. doi:10.1001/archneurol.2010.116

6. Rong Fu, PhD; Hyder A. Jinnah, MD, PhD, Different Phenotypes Among Lesch-Nyhan Variants: Clinical Reality or Limitation of Ascertainment?, Arch Neurol. 2011;68(2):264-271. doi:10.1001/archneurol.2010.360

7. Uros Hladnik, MD; William L. Nyhan, MD, PhD; Matteo Bertelli, MD, Variable Expression of HPRT Deficiency in 5 Members of a Family With the Same Mutation, Arch Neurol. 2008;65(9):1240-1243. doi:10.1001/ archneur.65.9.1240 
Lesch Nyhan Syndrome

8. Nyhan $\mathrm{WL}^{1}$, O’Neill JP${ }^{2}$, Jinnah $\mathrm{HA}^{3}$, Harris JC ${ }^{4}$, Lesch-Nyhan Syndrome, 2000 Sep 25 [updated 2014 May 15][PubMed]

9. William L Nyhan, MD, PhD, J Patrick O'Neill, PhD, Hyder A Jinnah, MD, PhD, and James C Harris, MD, LeschNyhan Syndrome, September 25, 2000; Last Update: May 15, 2014.[PubMed]

10. Scott Bell, Ilaria Kolobova, Liam Crapper, and Carl Ernst, Lesch-Nyhan Syndrome: Models, Theories, and Therapies, 2016 Sep 24. doi: 10.1159/000449296 [PubMed]

11. William L. Nyhan, MD, National organization for rare disorders, 501(c)(3) charity organization.

12. Dr Colin Tidy, Lesch-Nyhan Syndrome, 11 Dec 2014 [Patient Access]

13. Dr. H. A. Jinnah, Dr. J.E. Visser, Lesch Nyhan.org, HAJ \& JEV 2006-2018

14. Lesch-Nyhan syndrome, Wikipedia

15. NIH ;U.S.National Library of Medicine, Reviewed: February 2013, Published: May 22, 2018

Citation: K. M. D. L. Siriwardena, “Lesch Nyhan Syndrome”. American Research Journal of Pediatrics. 2018; 2(1): 1-9.

Copyright @ 2018 K. M. D. L. Siriwardena. This is an open access article distributed under the Creative Commons Attribution License, which permits unrestricted use, distribution, and reproduction in any medium, provided the original work is properly cited. 